M. Nishio

Nagoya Math. J.

Vol. 116 (1989), 163-179

\title{
THE WIENER CRITERION OF REGULAR POINTS FOR THE PARABOLIC OPERATOR OF ORDER $\alpha$
}

\author{
MASAHARU NISHIO
}

\section{$\S 1$. Introduction}

Let $R^{n+1}=R^{n} \times R$ denote the $(n+1)$-dimensional Euclidian space $(n \geqq 1)$. For $X \in R^{n+1}$, we write $X=(x, t)$ with $x \in R^{n}$ and $t \in R$. For an $\alpha$ with $0<\alpha<1$, we write

$$
L^{(\alpha)}=\frac{\partial}{\partial t}+(-\Delta)^{\alpha} \quad \text { and } \quad \tilde{L}^{(\alpha)}=-\frac{\partial}{\partial t}+(-\Delta)^{\alpha},
$$

where $\Delta$ is the Laplacian on $R^{n}$ and $(-\Delta)^{\alpha}$ is the $\alpha$-fractional power of $-\Delta$ on $R^{x}$.

The purpose of this paper is to establish the Wiener criterion of regular points for $L^{(\alpha)}$, where the division of the complement of a given open set is naturally determined by the form of the elementary solution $W^{(\alpha)}$ of $L^{(\alpha)}$ :

$$
W^{(\alpha)}(x, t)= \begin{cases}(2 \pi)^{-n} \int_{R^{n}} \exp \left(-t|\xi|^{2 \alpha}+i x \cdot \xi\right) d \xi & t>0 \\ 0 & t \leqq 0,\end{cases}
$$

where $x \cdot \xi$ denotes the inner product on $R^{n}$ and $|\xi|=(\xi \cdot \xi)^{1 / 2}$. Put $\phi_{\alpha}(|x|)$ $=W^{(\alpha)}(x, 1)$. Then $\phi_{\alpha}$ is decreasing on $[0, \infty), W^{(\alpha)}(x, t)=t^{-n / 2 \alpha} \phi_{\alpha}\left(t^{-1 / 2 \alpha}|x|\right)$ and $\phi_{\alpha}(r)$ is of order $r^{-n-2 \alpha}$ as $r \rightarrow \infty$. Let $X_{0}=\left(x_{0}, t_{0}\right) \in R^{n+1}$. For fixed $\lambda>1, \rho>1$ and non-negative integers $k, m$, we write

$$
A_{k, m}\left(X_{0}\right)=\left\{\left(x_{0}+x, t_{0}-t\right) ; t_{k+1} \leqq t \leqq t_{k}, r_{m} \leqq t^{-1 / 2 \alpha}|x| \leqq r_{m+1}\right\},
$$

where $t_{k}=\lambda^{-2 \alpha k / n}, r_{m}=\rho^{m /(n+2 \alpha)}$ for $m \geqq 1$ and $r_{0}=0$. The Wiener criterion of regular points for $L^{(\alpha)}$ is given by the following

THEOREM. Let $\Omega$ be an open set in $R^{n+1}$ and $X_{0} a$ boundary point of Q. Then $X_{0}$ is regular for the Dirichlet problem of $L^{(\alpha)}$ on $\Omega$ if and only if the following condition (w) is satisfied:

Received August 25, 1988. 


$$
\sum_{k, m=0}^{\infty} \lambda^{k} \rho^{-m} \operatorname{cap}^{(\alpha)}\left(A_{k, m}\left(X_{0}\right) \cap C \Omega\right)=\infty
$$

where $\operatorname{cap}^{(\alpha)}(\cdot)$ denotes the $\alpha$-parabolic capacity of $(\cdot)$.

From our theorem it follows immediately that a Poincaré type condition given in [6] is sufficient for regular points.

Furthermore our theorem gives another characterization of regular points; that is, a boundary point $X_{0}$ of $\Omega$ is regular if and only if

$$
\sum_{k=0}^{\infty} \rho^{k} \operatorname{cap}^{(\alpha)}\left(A_{k}\left(X_{0}\right) \cap C \Omega\right)=\infty,
$$

where $\rho>1$ and $A_{k}\left(X_{0}\right)=\left\{X \in R^{n+1} ; \rho^{k} \leqq W^{(\alpha)}\left(X_{0}-X\right) \leqq \rho^{k+1}\right\}$. For the heat equation, L.C. Evans and R.F. Gariepy obtained an analogous Wiener criterion of regular points (see [4], p. 295).

Finally we emphasize that the condition (w) is more convenient than $\left(\mathrm{w}^{\prime}\right)$.

The author would like to thank Professor M. Itô for his encouragement and helpful suggestions.

\section{§. $W^{(\alpha)}$-superharmonic functions and reduced functions}

Put $\tilde{W}^{(\alpha)}(x, t)=W^{(\alpha)}(x,-t)$. Then $\tilde{W}^{(\alpha)}$ is the elementary solution of $\tilde{L}^{(\alpha)}$. For any $s>0$, we define a positive measure $P_{s}^{(\alpha)}\left(\right.$ resp. $\left.\tilde{P}_{s}^{(\alpha)}\right)$ on $R^{n+1}$ by

$$
\begin{gathered}
\int f d P_{s}^{(\alpha)}=\int_{R^{n}} f(x, s) W^{(\alpha)}(x, s) d x \\
\left(\text { resp. } \int f d \tilde{P}_{s}^{(\alpha)}=\int_{R^{n}} f(x, s) \tilde{W}^{(\alpha)}(x, s) d x\right)
\end{gathered}
$$

for every $f \in C_{K}\left(R^{n+1}\right)$, and we put $P_{0}^{(\alpha)}=\tilde{P}_{0}^{(\alpha)}=\varepsilon$ (the Dirac measure at the origin). Here $C_{K}\left(R^{k}\right)$ denotes the usual topological vector space of all finite continuous functions on $R^{k}$ with compact support. Then $\int d P_{s}^{(\alpha)}=1$ for every $s \geqq 0$ and $\left(P_{s}^{(\alpha)}\right)_{s \geqq 0}\left(\right.$ resp. $\left.\left(\tilde{P}_{s}^{(\alpha)}\right)_{s \geqq 0}\right)$ is the convolution semi-group whose infinitesimal generator is $-L^{(\alpha)}$ (resp. $\left.-\tilde{L}^{(\alpha)}\right)$.

Definition 2.1. A non-negative function $u$ on $R^{n+1}$ is said to be $W^{(\alpha)}$-superharmonic (resp. $\tilde{W}^{(\alpha)}$-superharmonic) if the following two conditions are satisfied:

(1) $u$ is lower semi-continuous and $u<\infty$ a.e..

(2) For any $s>0, u \geqq P_{s}^{(\alpha)} * u$ (resp. $u \geqq \tilde{P}_{s}^{(\alpha)} * u$ ) on $R^{n+1}$. 
We denote by $S_{\alpha}$ (resp. $\tilde{S}_{\alpha}$ ) the set of all $W^{(\alpha)}$-superharmonic (resp. $\tilde{W}^{(\alpha)}$-superharmonic) functions and by $M_{\alpha}$ (resp. $\tilde{M}_{\alpha}$ ) the set of all positive Borel measures $\mu$ on $R^{n+1}$ such that $W^{(a)} \mu<\infty$ a.e. (resp. $\tilde{W}^{(\alpha)} \mu<\infty$ a.e.), where $W^{(\alpha)} \mu=W^{(\alpha)} * \mu$ (resp. $\tilde{W}^{(\alpha)} \mu=\tilde{W}^{(\alpha)} * \mu$ ). Evidently $W^{(\alpha)} \mu \in S_{\alpha}$ (resp. $\tilde{W}^{(\alpha)} \mu \in \tilde{S}_{\alpha}$ ) for every $\mu \in M_{\alpha}$ (resp. $\mu \in \tilde{M}_{\alpha}$ ).

Since $\lim _{s \rightarrow 0} P_{s}^{(\alpha)}=\varepsilon$ (vaguely), we have the following

Remark 2.2. Let $u \in S_{\alpha}$. Then for any $(x, t) \in R^{n+1}$,

$$
\begin{aligned}
u(x, t) & =\lim _{\tau \rightarrow 0} \frac{1}{\tau} \int_{0}^{\tau} P_{s}^{(\alpha)} * u(x, t) d s \\
& =\lim _{\tau \rightarrow 0} \frac{1}{\tau} \int_{0}^{\tau} \int_{R^{n}} u(x-y, t-s) W^{(\alpha)}(y, s) d y d s .
\end{aligned}
$$

Proposition 2.3 ([6], Proposition 2.10). Let $u \in S_{\alpha}$ (resp. $\left.\tilde{S}_{\alpha}\right)$. Then there exists uniquely determined $(\mu, c) \in M_{\alpha} \times[0, \infty)$ (resp. $\left.\tilde{M}_{\alpha} \times[0, \infty)\right)$ such that

$$
u=W^{(\alpha)} \mu+c \quad\left(\text { resp. } u=\tilde{W}^{(\alpha)} \mu+c\right) .
$$

The measure $\mu$ in the above proposition is called the associated measure of $u$.

Corollary 2.4. Let $u \in S_{\alpha}$ and let $\mu$ be the associated measure of $u$. If $u \leqq W^{(\alpha)} \nu$ for some $\nu \in M_{\alpha}$, then $u=W^{(\alpha)} \mu$.

Let $u \in S_{\alpha}$ (resp. $u \in \tilde{S}_{\alpha}$ ). We say that $u$ is a $W^{(\alpha)}$-potential (resp. $\tilde{W}^{(\alpha)}$-potential) if $u=W^{(\alpha)} \mu$ (resp. $u=\tilde{W}^{(\alpha)} \mu$ ), where $\mu$ is the associated measure of $u$.

For a finite continuous $u \in S_{\alpha}$ and a compact set $K$ in $R^{n+1}$, we put

$$
Q_{K}^{(\alpha)} u(Y)=\inf \left\{v(Y) ; v \in S_{\alpha}, v \geqq u \text { on } K\right\}
$$

and

$$
R_{K}^{(\alpha)} u(X)=\liminf _{Y \rightarrow X} Q_{K}^{(\alpha)} u(Y) .
$$

Furthermore for any $u \in S_{\alpha}$ and any set $A$ in $R^{n+1}$, we write $R_{A}^{(\alpha)} u(X)$

$$
=\sup \left\{R_{K}^{(\alpha)} v(X) ; v \in S_{\alpha}, v \leqq u, v \text { : finite continuous, } K \text { : compact } \subset A\right\} .
$$

For the justification of the above definition, we need to remark that for any $u \in S_{\alpha}$, there exists $\left(u_{m}\right)_{m=1}^{\infty}$ of finite continuous $W^{(\alpha)}$-superharmonic 
functions which converges increasingly to $u$ with $m \uparrow \infty$ (see [6], Lemma 2.4, (2)). We have $R_{A}^{(\alpha)} u \in S_{\alpha}$ and call $R_{A}^{(\alpha)} u$ the $W^{(\alpha)}$-reduced function of $u$ to $A$. Similarly we define the $\tilde{W}^{(\alpha)}$-reduced function $\tilde{R}_{A}^{(\alpha)} u \in \tilde{S}_{\alpha}$ of $u\left(u \in \tilde{S}_{\alpha}\right)$ to $A$.

Proposition 2.5. Let $u \in S_{\alpha}$ and let $A$ be a set in $R^{n+1}$. Then we have:

(1) If $A \subset R^{n} \times(a, \infty)$ with some $a \in R$, then $R_{A}^{(\alpha)} u$ is a $W^{(\alpha)}$-potential.

(2) $u=R_{A}^{(\alpha)} u$ on $A^{i}$, where $A^{i}$ denotes the interior of $A$.

(3) The associated measure of $R_{A}^{(\alpha)} u$ is supported by $\bar{A}$.

In fact, since $\int W^{(\alpha)}(x-y, t-a) d y=1$ on $R^{n} \times(a, \infty)$, Proposition 2.3 shows that $u \leqq W^{(\alpha)} \nu$ on $A$ for some $\nu \in M_{\alpha}$, and hence Corollary 2.4 gives (1). The statements (2) and (3) are already known in [6] (see Remark 3.1, (2) and Proposition 3.7).

The analogous result to $\tilde{W}^{(\alpha)}$-reduced functions also holds.

Proposition 2.6 ([6], Lemmas 2.7 and 3.5). Let $u \in S_{\alpha}$ and let $K$ be a compact set in $R^{n+1}$ and $\left(\omega_{m}\right)_{m=1}^{\infty} a$ sequence of relatively compact open sets in $R^{n+1}$ with $\overline{\omega_{m+1}} \subset \omega_{m}$ and $\cap_{m=1}^{\infty} \omega_{m}=K$. If $u$ is finite continuous on $a$ certain neighborhood of $K$, then

$$
\lim _{m \rightarrow \infty} R_{\omega_{m}}^{(\alpha)} u=R_{K}^{(\alpha)} u \quad \text { a.e. } \quad \text { and } \quad \lim _{m \rightarrow \infty} \mu_{\omega_{m}}=\mu_{K} \text { (vaguely), }
$$

where $\mu_{\omega_{m}}$ and $\mu_{K}$ are the associated measures of $R_{\omega_{m}}^{(\alpha)} u$ and of $R_{K}^{(\alpha)} u$, respectively.

Let $u=W^{(\alpha)} \mu$ (resp. $u=\tilde{W}^{(\alpha)} \mu$ ) with $\mu \in M_{\alpha}$ (resp. $\mu \in \tilde{M}_{\alpha}$ ) and let $A$ be a set in $R^{n+1}$. Then $R_{A}^{(\alpha)} u$ (resp. $\tilde{R}_{A}^{(\alpha)} u$ ) is a $W^{(\alpha)}$-potential (resp. $\tilde{W}^{(\alpha)}$ potential) and the associated measure $\mu_{A}^{\prime}$ (resp. $\mu_{A}^{\prime \prime}$ ) of $R_{A}^{(\alpha)} u$ (resp. $\tilde{R}_{A}^{(\alpha)} u$ ) is called the $W^{(\alpha)}$-swept-out (resp. $\tilde{W}^{(\alpha)}$-swept-out) measure of $\mu$ to $A$.

Proposition 2.7. Let $\mu, \nu \in M_{\alpha}$. If $W^{(\alpha)} \mu \leqq W^{(\alpha)} \nu$ on a certain open set containing $\operatorname{supp}[\mu]$, then $W^{(\alpha)} \mu \leqq W^{(\alpha)} \nu$ on $R^{n+1}$ and $\int d \mu \leqq \int d \nu$. Here $\operatorname{supp}[\mu]$ denotes the suppcrt of $\mu$.

Proof. The first required inequality follows from Proposition 3.11 in [6]. To show our second required inequality, we may assume that supp [ $\mu$ ] is compact. Let $\omega$ be a relatively compact open set in $R^{n+1}$ with $\omega \supset$ $\operatorname{supp}[\mu]$ and $\lambda$ the associated measure of $\tilde{R}_{\omega}^{(\alpha)} 1$. Then Corollary 2.4 and Proposition 2.5 , (2) give $\tilde{W}^{(\alpha)} \lambda=1$ on $\omega$, so that 


$$
\int d \mu=\int \tilde{W}^{(\alpha)} \lambda d \mu=\int W^{(\alpha)} \mu d \lambda \leqq \int W^{(\alpha)} \nu d \lambda=\int \tilde{W}^{(\alpha)} \lambda d \nu \leqq \int d \nu .
$$

The analogous statement to $\tilde{W}^{(\alpha)}$-potentials also holds. In particular, we have the following

Corollary 2.8. Let $\mu \in M_{\alpha}$ and let $A$ be a set in $R^{n+1}$. Then $\int d \mu_{A}^{\prime} \leqq$ $\int d \mu$, and if $\operatorname{supp}[\mu] \subset A^{i}$, then $\mu_{A}^{\prime}=\mu$.

Proposition 2.9 ([6], Proposition 3.8). Let $\mu \in M_{\alpha}$ and $\nu \in \tilde{M}_{\alpha}$. Then for a set $A$ in $R^{n+1}$, we have

$$
\int W^{(\alpha)} \mu_{A}^{\prime} d \nu=\int W^{(\alpha)} \mu d \nu_{A}^{\prime \prime} \quad \text { and } \quad W^{(\alpha)} \mu_{A}^{\prime}(X)=\int W^{(\alpha)} \varepsilon_{Y, A}^{\prime}(X) d \mu(Y),
$$

where we denote by $\varepsilon_{Y}$ and by $\varepsilon_{Y, A}^{\prime}$ the Dirac measure at $Y$ and the $W^{(\alpha)}$ swept-out measure of $\varepsilon_{Y}$ to $A$, respectively.

Proposition 2.9 gives the following

Corollary 2.10. Let $A$ be a set in $R^{n+1}$ and let $u, v \in S_{\alpha}$. Then

$$
R_{A}^{(\alpha)}(u+v)=R_{A}^{(\alpha)} u+R_{A}^{(\alpha)} v .
$$

Proof. We may assume that $A$ is compact (see the definition of $R_{A}^{(\alpha)} u$ ). Let $\omega$ be a relatively compact open set with $\omega \supset A$. Since $R_{\omega}^{(\alpha)} u$ and $R_{\omega}^{(\alpha)} v$ are $W^{(\alpha)}$-potentials, Proposition 2.9 gives

$$
R_{A}^{(\alpha)}\left(R_{\omega}^{(\alpha)} u+R_{\omega}^{(\alpha)} v\right)=R_{A}^{(\alpha)} R_{\omega}^{(\alpha)} u+R_{A}^{(\alpha)} R_{\omega}^{(\alpha)} v .
$$

This and Proposition 2.5, (2) imply our required equality.

Definition 2.11. Let $\Omega$ be an open set in $R^{n+1}$ and $X_{0}$ a boundary point of $\Omega$. Then $X_{0}$ is said to be regular (for the Dirichlet problem of $\left.L^{(\alpha)}\right)$ on $\Omega$ if

$$
\lim _{X \in \Omega, X \rightarrow X_{0}} \varepsilon_{X, C \Omega}^{\prime \prime}=\varepsilon_{X_{0}} \quad \text { (vaguely). }
$$

Proposition 2.12 ([6], Propositions 4.1, 4.2). Let $\Omega$ be an open set, $X_{0}$ a boundary point of $\Omega$ and $K$ a compact neighborhood of $X_{0}$. Then $X_{0}$ is regular on $\Omega$ if and only if $R_{K \cap C \Omega}^{(\alpha)} 1\left(X_{0}\right)=1$.

For irregular points, the following remarkable statement holds.

Proposition 2.13. Let $E$ be a closed set in $R^{n+1}$ and $X_{0} \in R^{n+1}$. If 
$R_{E}^{(\alpha)} 1\left(X_{0}\right)<1$, then

$$
\inf \left\{R_{E \cap U}^{(\alpha)} 1\left(X_{0}\right) ; U: \text { neighborhood of } X_{0}\right\}=0 .
$$

For the proof, we shall use the following

Lemma 2.14. Let $u \in S_{\alpha}$ and let $K$ be a compact set and $A$ a set in $R^{n+1}$ with $K \subset A$. Denote by $\mu_{K}$ and $\mu_{A}$ the associated measures of $R_{K}^{(\alpha)} u$ and of $R_{A}^{(\alpha)} u$, respectively. If $u$ is finite continuous on a certain neighborhood of $K$ and $R_{A}^{(\alpha)} u=W^{(\alpha)} \mu_{A}$ on $R^{n+1}$, then

$$
\mu_{K}=\left.\mu_{A}\right|_{K}+\left(\left.\mu_{A}\right|_{C K}\right)_{K}^{\prime} \cdot{ }^{1)}
$$

Proof. By Proposition 2.5, (1), $R_{K}^{(\alpha)} u=W^{(\alpha)} \mu_{K}$. Take a sequence $\left(\omega_{m}\right)_{m=1}^{\infty}$ of relatively compact open sets in $R^{n+1}$ such that $\overline{\omega_{m+1}} \subset \omega_{m}$ and $\cap_{m=1}^{\infty} \omega_{m}=K$. Since $\operatorname{supp}\left[\mu_{K}\right] \subset K \subset \omega_{m}$, we have $\left(\mu_{K}\right)_{\omega_{m}}^{\prime}=\mu_{K}$ (see Corollary 2.8). Since $u \geqq W^{(\alpha)} \mu_{A} \geqq W^{(\alpha)} \mu_{K}$ on $R^{n+1}$,

$$
R_{\omega_{m}}^{(\alpha)} u \geqq R_{\omega_{m}}^{(\alpha)} W^{(\alpha)} \mu_{A} \geqq W^{(\alpha)} \mu_{K} \quad \text { on } R^{n+1},
$$

and by Proposition 2.6, we have $\lim _{m \rightarrow \infty} R_{\omega_{m}}^{(\alpha)} W^{(\alpha)} \mu_{A}=W^{(\alpha)} \mu_{K}$ a.e. By Proposition 2.9 and Corollary 2.8, we have

$$
\begin{aligned}
W^{(\alpha)}\left(\mu_{A}\right)_{\omega_{m}}^{\prime}(X) & =W^{(\alpha)}\left(\left.\mu_{A}\right|_{K}\right)_{\omega_{m}}^{\prime}(X)+W^{(\alpha)}\left(\left.\mu_{A}\right|_{C K}\right)_{\omega_{m}}^{\prime}(X) \\
& =\left.W^{(\alpha)} \mu_{A}\right|_{K}(X)+\left.\int W^{(\alpha)} \hat{\varepsilon}_{Y, \omega_{m}}^{\prime}(X) d \mu_{A}\right|_{C K}(Y) \\
& =\left.W^{(\alpha)} \mu_{A}\right|_{K}(X)+\sum_{k=0}^{\infty} W^{(\alpha)}\left(\left.\mu_{A}\right|_{\omega_{k} \cap \omega_{\omega_{k+1}}}\right)_{\omega_{m}}^{\prime}(X),
\end{aligned}
$$

where $\omega_{0}=R^{n+1}$. Since $W^{(\alpha)}\left(\left.\mu_{A}\right|_{\omega_{k} \cap \omega_{\omega_{k+1}}}\right)$ is finite continuous on $\omega_{k+1}$, Proposition 2.6 gives

$$
\lim _{m \rightarrow \infty} W^{(\alpha)}\left(\left.\mu_{A}\right|_{\omega_{k} \cap \omega_{\omega_{k+1}}}\right)_{\omega_{m}}^{\prime}=W^{(\alpha)}\left(\left.\mu_{A}\right|_{\omega_{k} \cap \omega_{\omega_{k+1}}}\right)_{K}^{\prime} \quad \text { a.e. }
$$

for every $k \geqq 0$, which implies

$$
\begin{aligned}
W^{(\alpha)} \mu_{K} & =\left.W^{(\alpha)} \mu_{A}\right|_{K}+\sum_{k=0}^{\infty} W^{(\alpha)}\left(\left.\mu_{A}\right|_{\omega_{k} \cap \omega_{\omega_{k+1}}}\right)_{K}^{\prime} \\
& =\left.W^{(\alpha)} \mu_{A}\right|_{K}+W^{(\alpha)}\left(\left.\mu_{A}\right|_{C K}\right)_{K}^{\prime} \quad \text { a.e., }
\end{aligned}
$$

so that

$$
W^{(\alpha)} \mu_{K}=W^{(\alpha)}\left(\left.\mu_{A}\right|_{K}+\left(\left.\mu_{A}\right|_{C K}\right)_{K}^{\prime}\right) \quad \text { on } R^{n+1}
$$

1) For a Borel measure $\mu$ and a Borel set $A$ in $R^{n+1}$, we denote by $\left.\mu\right|_{A}$ the Borel measure defined by $\left.\mu\right|_{A}(E)=\mu(E \cap A)$ for every Borel set $E$ in $R^{n+1}$. 
(see Remark 2.2). Hence by Proposition 2.3, our required equality holds.

Proof of Proposition 2.13. We may assume that $E$ is compact, $X_{0}=0$ (the origin) and that $R_{E}^{(\alpha)} 1(0)>0$. Let $\mu$ be the associated measure of $R_{E}^{(\alpha)} 1$. Then $R_{E}^{(\alpha)} 1=W^{(\alpha)} \mu$. For any $\tau>0$, we write $\mu_{\tau, 1}=\left.\mu\right|_{R^{n} \times(-\tau, \infty)}$ and $\mu_{\tau, 2}=\left.\mu\right|_{R^{n} \times(-\infty,-\tau]}$. Put

$$
\gamma=R_{E}^{(\alpha)} 1(0) \text { and } \beta=\inf \left\{R_{E \cap U}^{(\alpha)} 1(0) ; U \text { : neighborhood of } 0\right\} .
$$

Assume $\beta>0$. Then there exists a compact neighborhood $U$ of 0 such that

$$
R_{E \cap U}^{(\alpha)} 1(0)<\frac{\beta}{2}\left(1+\frac{1}{\gamma}\right)
$$

Putting $\delta=\beta(1-\gamma) / 4$, we choose $\tau>0$ such that $W^{(\alpha)} \mu_{\tau, 1}(0)<\delta$. Set $F$ $=R^{n} \times(-\infty,-\tau]$,

$$
c=\inf \left\{W^{(\alpha)} \varepsilon_{Y}(0) ; Y=(y, s) \in E, s \leqq-\tau\right\}>0 \text { and } \delta^{\prime}=\frac{c(1-\gamma)}{2(1+\gamma)} \text {. }
$$

Then there exists a compact neighborhood $V$ of 0 such that $V \subset U \cap C F$ and for any $X \in V$ and $Y \in E \cap F$,

$$
W^{(\alpha)} \varepsilon_{Y}(X) \leqq\left(1+\frac{\delta^{\prime}}{c}\right) W^{(\alpha)} \varepsilon_{Y}(0) .
$$

Denoting by $\varepsilon_{Y, E_{n} V}^{\prime}$ the associated measure of $R_{E_{n}}^{(\alpha)} W^{(\alpha)} \varepsilon_{Y}$, we have

$$
\begin{aligned}
W^{(\alpha)} \varepsilon_{Y, E \cap V}^{\prime}(0) & \leqq\left(1+\frac{\delta^{\prime}}{c}\right) W^{(\alpha)} \varepsilon_{Y}(0) R_{E \cap V}^{(\alpha)} 1(0) \\
& \leqq \frac{\beta}{2}\left(1+\frac{1}{\gamma}\right)\left(1+\frac{\delta^{\prime}}{c}\right) W^{(\alpha)} \varepsilon_{Y}(0)
\end{aligned}
$$

for every $Y \in E \cap F$, so that by Proposition 2.9, we have

$$
W^{(\alpha)}\left(\mu_{\tau, 2}\right)_{E \cap V}^{\prime}(0) \leqq \frac{(3+\gamma) \beta}{4 \gamma} W^{(\alpha)} \mu_{\tau, 2}(0)
$$

Let $\nu$ be the associated measure of $R_{E \cap(V \cup F)}^{(\alpha)} 1$. Since $\left(\nu_{r, 1}\right)_{E \cap F}^{\prime}=\left(\mu_{\tau, 1}\right)_{E \cap F}^{\prime}=$ $0,{ }^{2)}$ Lemma 2.14 shows that $\nu_{\tau, 2}$ and $\mu_{\tau, 2}$ are equal to the associated measure of $R_{E \cap F}^{(\alpha)} 1$, that is, $\nu_{\tau, 2}=\mu_{\tau, 2}$, which implies $W^{(\alpha)} \nu_{\tau, 1} \leqq W^{(\alpha)} \mu_{\tau, 1}$ on $R^{n+1}$. Hence Lemma 2.14 gives

2) The notation $\nu_{\tau, i}$ is the same as $\mu_{\nu, i}(i=1,2)$. 


$$
\begin{aligned}
R_{E \cap V}^{(\alpha)} 1(0) & =W^{(\alpha)} \nu_{\tau, 1}(0)+W^{(\alpha)}\left(\nu_{\tau, 2}\right)_{E \cap V}^{\prime}(0) \\
& \leqq W^{(\alpha)} \mu_{\tau, 1}(0)+W^{(\alpha)}\left(\mu_{\tau, 2}\right)_{E \cap V}^{\prime}(0) \\
& <\delta+\frac{(3+\gamma) \beta}{4 \gamma} W^{(\alpha)} \mu(0)=\beta
\end{aligned}
$$

which is a contradiction. Thus Proposition 2.13 is shown.

\section{$\S 3$. The $\alpha$-parabolic capacity}

Let $K$ be a compact set in $R^{n+1}$ and put

$$
\Phi_{K}^{(\alpha)}=\left\{\mu \in M_{\alpha} ; \operatorname{supp}[\mu] \subset K, W^{(\alpha)} \mu \leqq 1 \text { on } R^{n+1}\right\} .
$$

LEMma 3.1. For a compact set $K$ in $R^{n+1}$, we denote by $\mu_{K}$ and by $\nu_{K}$ the associated measures of $R_{K}^{(\alpha)} 1$ and of $\tilde{R}_{K}^{(\alpha)} 1$, respectively. Then we have

$$
W^{(\alpha)} \mu_{K}(X)=\sup \left\{W^{(\alpha)} \mu(X) ; \mu \in \Phi_{K}^{(\alpha)}\right\}
$$

and

$$
\int d \mu_{K}=\int d \nu_{K}=\sup \left\{\int d \mu ; \mu \in \Phi_{K}^{(\alpha)}\right\}
$$

Proof. Let $\mu \in \Phi_{K}^{(\alpha)}$. Then for any relatively compact open set $\omega \supset K$, Proposition 2.7 shows that $W^{(\alpha)} \mu \leqq R_{\omega}^{(\alpha)} 1$ on $R^{n+1}$ (see also Proposition 2.5, (1), (2)). By Proposition 2.6, we have $W^{(\alpha)} \mu \leqq W^{(\alpha)} \mu_{K}$ a.e., and hence $W^{(\alpha)} \mu \leqq W^{(\alpha)} \mu_{K}$ on $R^{n+1}$ (see Remark 2.2). On the other hand, by Proposition 2.5, (3), $\mu_{K} \in \Phi_{K}^{(\alpha)}$. These imply the equality (1), and by Proposition 2.7, we have $\int d \mu_{K}=\sup \left\{\int d \mu ; \mu \in \Phi_{K}^{(\alpha)}\right\}$. Let $\omega$ be a relatively compact open set in $R^{n+1}$, and denote by $\mu_{\omega}$ and by $\nu_{\omega}$ the associated measures of $R_{\omega}^{(\alpha)} 1$ and of $\tilde{R}_{\omega}^{(\alpha)} 1$, respectively. By Proposition 2.5 , (1), (2),

$$
W^{(\alpha)} \mu_{K}=R_{K}^{(\alpha)} W^{(\alpha)} \mu_{\omega} \quad \text { and } \quad \tilde{W}^{(\alpha)} \nu_{K}=\tilde{R}_{K}^{(\alpha)} \tilde{W}^{(\alpha)} \nu_{\omega} \quad \text { on } \quad R^{n+1} .
$$

Since $W^{(\alpha)} \mu_{\omega}=\tilde{W}^{(\alpha)} \nu_{\omega}=1$ on $\omega$, Proposition 2.9 shows the equality $\int d \mu_{R}$ $=\int d \nu_{K}$. Thus Lemma 3.1 is shown.

We shall define the $\alpha$-parabolic capacity in the usual manner.

Definition 3.2. For a compact set $K$ in $R^{n+1}$, we put

$$
\operatorname{cap}^{(\alpha)}(K)=\sup \left\{\int d \mu ; \mu \in \Phi_{K}^{(\alpha)}\right\}
$$

Then we have the following 
Proposition 3.3. Denoto by $\mathscr{K}$ the totality of compact sets in $R^{n+1}$. Then $\operatorname{cap}^{(\alpha)}(K)$ defined on $\mathscr{K}$ is a Choquet capacity.

Proof. Evidently $\operatorname{cap}^{(\alpha)}(\phi)=0$ and $\operatorname{cap}^{(\alpha)}(K)$ is increasing. Let $K \in \mathscr{K}$. Then Proposition 2.6 shows that for any $\delta>0$, there exists a relatively compact open set $\omega_{\delta}$ with $\omega_{\delta} \supset K$ satisfying $\int d \mu_{K}>\int d \mu_{\omega_{\delta}}-\delta$, where $\mu_{K}$ and $\mu_{\omega_{\delta}}$ are the associated measures of $R_{K}^{(\alpha)} 1$ and of $R_{\omega_{\delta}}^{(\alpha)} 1$, respectively. By Lemma 3.1, we obtain that $\operatorname{cap}^{(\alpha)}(K)$ is right continuous. We shall show that $\operatorname{cap}^{(\alpha)}(K)$ is strongly subadditive. Let $K_{1}, K_{2} \in \mathscr{K}$ and let $\omega_{1}$ and $\omega_{2}$ be relatively compact open sets in $R^{n+1}$ with $\omega_{1} \supset K_{1}$ and $\omega_{2} \supset K_{2}$. By Proposition 2.5, (2), we have

$$
R_{K_{1} \cup K_{2}}^{(\alpha)} 1+R_{K_{1} \cap K_{2}}^{(\alpha)} 1 \leqq R_{\omega_{1}}^{(\alpha)} 1+R_{\omega_{2}}^{(\alpha)} 1 \quad \text { on } \omega_{1} \cup \omega_{2} .
$$

By Proposition 2.7, Lemma 3.1 and the right continuity of $\operatorname{cap}^{(\alpha)}(K)$, we have

$$
\operatorname{cap}^{(\alpha)}\left(K_{1} \cup K_{2}\right)+\operatorname{cap}^{(\alpha)}\left(K_{1} \cap K_{2}\right) \leqq \operatorname{cap}^{(\alpha)}\left(K_{1}\right)+\operatorname{cap}^{(\alpha)}\left(K_{2}\right),
$$

which shows the strong subadditivity of $\operatorname{cap}^{(\alpha)}(K)$. Thus $\operatorname{cap}^{(\alpha)}(K)$ is a Choquet capacity.

Recalling the definition of $W^{(\alpha)}$-reduced functions and the above proof, we see the following

Remark 3.4. Let $F_{1}$ and $F_{2}$ be two closed sets in $R^{n+1}$. Then

$$
R_{F_{1} \cup F_{2}}^{(\alpha)} 1+R_{F_{1} \cap F_{2}}^{(\alpha)} 1 \leqq R_{F_{1}}^{(\alpha)} 1+R_{F_{2}}^{(\alpha)} 1 \quad \text { on } R^{n+1} .
$$

Definition 3.5. For a set $A$ in $R^{n+1}$, we put

$$
\operatorname{cap}_{i}^{(\alpha)}(A)=\sup \left\{\operatorname{cap}^{(\alpha)}(K) ; A \supset K \in \mathscr{K}\right\}
$$

and

$$
\operatorname{cap}_{e}^{(\alpha)}(A)=\inf \left\{\operatorname{cap}_{i}^{(\alpha)}(\omega): A \subset \omega: \text { open set }\right\} .
$$

We say that $A$ is capacitable if $\operatorname{cap}_{i}^{(\alpha)}(A)=\operatorname{cap}_{e}^{(\alpha)}(A)$. In the case that $A$ is capacitable, we write $\operatorname{cap}^{(\alpha)}(A)=\operatorname{cap}_{i}^{(\alpha)}(A)$ and call $\operatorname{cap}^{(\alpha)}(A)$ the $\alpha$ parabolic capacity of $A$.

By Proposition 3.3 and the Choquet theory (cf. [2], p. 158), every analytic set in $R^{n+1}$ is capacitable.

Noticing the form of $W^{(\alpha)}$ and Proposition 2.3, we see that for any $\lambda>0, S_{\alpha}$ is stable for the $\alpha$-parabolic dilation $\tau_{\lambda}:(x, t) \rightarrow\left(\lambda x, \lambda^{2 \alpha} t\right)$ on $R^{n+1}$, that is, for any $u \in S_{\alpha}, u \circ \tau_{\lambda} \in S_{\alpha}$. Furthermore for any compact set $K$ in $R^{n+1}$, we have 


$$
W^{(\alpha)} \mu_{K_{\lambda}}\left(\tau_{\lambda}(X)\right)=W^{(\alpha)} \mu_{K}(X),
$$

where $K_{\lambda}=\left\{\tau_{\lambda}(X) ; X \in K\right\}$ and $\mu_{K}$ and $\mu_{K_{\lambda}}$ denote the associated measures of $R_{K}^{(\alpha)} 1$ and of $R_{K_{\lambda}}^{(\alpha)} 1$, respectively. Therefore we have the following

Proposition 3.6. For any compact set $K$ in $R^{n+1}$ and $\lambda>0$, we have

$$
\operatorname{cap}^{(\alpha)}\left(K_{\lambda}\right)=\lambda^{n} \operatorname{cap}^{(\alpha)}(K) \text {. }
$$

Remark 3.7. Let $B$ be a Borel set of $R^{n} \times\{a\}$, where $a \in R$. Then the thermal capacity of $B^{3)}$ is equal to the $n$-dimensional Lebesgue measure of $B$ (see [7], p. 355). Similarly the $\alpha$-parabolic capacity of $B$ is also equal to the $n$-dimensional Lebesgue measure of $B$.

\section{§4. The Wiener criterion of regular points}

First we shall show the following

LEMma 4.1. Put $\phi_{\alpha}(|x|)=W^{(\alpha)}(x, 1)$. For any $r_{0}>0$, there exists $a$ constant $C\left(r_{0}\right)>1$ such that for $r_{0} \leqq r<\infty$,

$$
C\left(r_{0}\right)^{-1} r^{-n-2 \alpha} \leqq \phi_{\alpha}(r) \leqq C\left(r_{0}\right) r^{-n-2 \alpha} .
$$

Proof. Put $\psi_{\alpha}(t)=\int_{R^{n}} W^{(\alpha)}(x, t) d \nu(x)$, where $\nu$ is the uniform measure on $\left\{x \in R^{n} ;|x|=1\right\}$ with $\int d \nu=1$. Then $\psi_{\alpha}$ is positive and finite continuous on $(0, \infty)$. Hence it suffices to show that $0<\lim _{t \downarrow 0}(d / d t) \psi_{\alpha}(t)<\infty$, because $\phi_{\alpha}(r)=r^{-n} \psi_{\alpha}\left(r^{-2 \alpha}\right)$. In the proof of Lemma 2.1 in [6], we already obtained the equality

$$
\frac{d}{d t} \psi_{\alpha}(t)=-(2 \pi)^{-n / 2} \int_{0}^{\infty} \int_{R^{n}}|\xi|^{2 \alpha} \exp \left(-s|\xi|^{2}\right) \hat{\nu}(\xi) d \xi d \sigma_{t}^{\alpha}(s),
$$

where $\left(\sigma_{t}^{\alpha}\right)_{t \geqq 0}$ is the one sided stable semi-group of order $\alpha$ on $R$ (see [1], p. 74) and where $\hat{\nu}$ denotes the Fourier transform of $\nu$. Since $\sigma_{t}^{\alpha}$ converges vaguely to $\varepsilon$ as $t \downarrow 0$, it suffices to show that

$$
0<\lim _{s \downarrow 0}\left(-(2 \pi)^{-n / 2} \int_{R^{n}}|\xi|^{2 \alpha} \exp \left(-s|\xi|^{2}\right) \hat{\nu}(\xi) d \xi\right)<\infty .
$$

If $\alpha=1 / 2$, then

$$
W^{(\alpha)}(x, t)=\Gamma\left(\frac{n+1}{2}\right) t\left\{\pi\left(|x|^{2}+t^{2}\right)\right\}^{-(n+1) / 2}
$$

3) This means the capacity of $B$ with respect to $(\partial / \partial t)-\Delta$ defined in the same manner as the $\alpha$-parabolic capacity of $B$. 
(see [1], p. 74), and hence we may assume $\alpha \neq 1 / 2$. Let $\left(g_{s}\right)_{s \geqq 0}$ be the Gaussian semi-group on $R^{n}$ and choose a function $f$ on $R^{n}$ of class $C^{\infty}$ satisfying $0 \leqq f \leqq 1, \operatorname{supp}[f] \subset\left\{x \in R^{n} ;|x|<1\right\}$ and $f=1$ on a certain neighborhood of the origin 0 . In the proof of Lemma 2.1 in [6], we already obtained

$$
\begin{aligned}
(2 \pi)^{-n / 2} & \int_{R^{n}}|\xi|^{2 \alpha} \exp \left(-s|\xi|^{2}\right) \hat{\nu}(\xi) d \xi \\
= & C_{n, \alpha-1}\left(f(x)|x|^{-n-2 \alpha+2}\right) *\left(\Delta g_{s}\right) * \nu(0) \\
& +C_{n, \alpha-1}\left(\Delta\left((1-f(x))|x|^{-n-2 \alpha+2}\right)\right) * g_{s} * \nu(0),
\end{aligned}
$$

where

$$
C_{n, \alpha-1}=-4^{\alpha-1} \pi^{-n / 2} \frac{\Gamma((n+2 \alpha-2) / 2)}{\Gamma(1-\alpha)}
$$

Having

$$
\Delta\left((1-f(x))|x|^{-n-2 \alpha+2}\right)=2 \alpha(n+2 \alpha-2) \quad \text { on }\left\{x \in R^{n} ;|x|=1\right\}
$$

and

$$
\left(f(x)|x|^{-n-2 a+2}\right) * \nu=0
$$

on a certain neighborhood of 0 in $R^{n}$, we conclude

$$
\lim _{s \downarrow 0}\left(-(2 \pi)^{-n / 2} \int_{R^{n}}|\xi|^{2 \alpha} \exp \left(-s|\xi|^{2}\right) \hat{\nu}(\xi) d \xi\right)=\alpha 4^{\alpha} \pi^{-n / 2} \frac{\Gamma((n+2 \alpha) / 2)}{\Gamma(1-\alpha)} .
$$

Thus Lemma 4.1 is shown.

Our main theorem is the following

THEOREM. Let $\Omega$ be an open set in $R^{n+1}, X_{0}$ a boundary point of $\Omega$, $\lambda>1$ and $\rho>1$. Then $X_{0}$ is regular for the Dirichlet problem of $L^{(\alpha)}$ on $\Omega$ if and only if the following condition (w) holds:

$$
w_{\lambda, \rho}:=\sum_{k, m=0}^{\infty} \lambda^{k} \rho^{-m} \operatorname{cap}^{(\alpha)}\left(A_{k, m}\left(X_{0}\right) \cap C \Omega\right)=\infty,
$$

where

$$
A_{k, m}\left(X_{0}\right)=\left\{X_{0}+(x,-t) ; t_{k+1} \leqq t \leqq t_{k}, r_{m} \leqq t^{-1 / 2 \alpha}|x| \leqq r_{m+1}\right\},
$$

with $t_{k}=\lambda^{-2 \alpha k / n}, r_{m}=\rho^{m /(n+2 \alpha)}$ for $m \geqq 1$ and $r_{0}=0$.

Since $W^{(\alpha)}(x, t)=t^{-n / 2 \alpha} \phi_{\alpha}\left(t^{-1 / 2 \alpha}|x|\right)$, we have the following

Remark 4.2. For any $Y \in A_{k, m}\left(X_{0}\right)$, we have 


$$
C_{0}^{-1} \lambda^{k} \rho^{-m-1} \leqq W^{(\alpha)} \varepsilon_{Y}\left(X_{0}\right) \leqq C_{0} \lambda^{k+1} \rho^{-m},
$$

where $C_{0}$ is a constant depending only on $n$ and $\alpha$.

Proof of Theorem. By using a suitable translation if necessary, we may assume that $X_{0}$ is the origin 0. Put $A_{k, m}=A_{k, m}(0)$ and $E_{k, m}=A_{k, m}$ $\cap C \Omega$. By the strong subadditivity of $\operatorname{cap}^{(\alpha)}(K)$ on $\mathscr{K}$, it suffices to prove the following

(*) 0 is regular for the Dirichlet problem of $L^{(\alpha)}$ on $\Omega$ if and only if the condition (w) holds for $\lambda=\rho$ and $\rho$ (that is, $w_{\rho, \rho}=\infty$ ).

In fact, if $\lambda_{1}$ and $\lambda_{2}$ satisfiy $1<\lambda_{1}<\lambda_{2}<\lambda_{1}^{l}$ for some integer $l$, then we have

$$
\left(2 \lambda_{1}\right)^{-1} w_{\lambda_{2}, \rho} \leqq w_{\lambda_{1}, \rho} \leqq \lambda_{2}(l+1) w_{\lambda_{2}, \rho}
$$

for every $\rho>1$.

Assume that the condition (w) for $\lambda=\rho$ and $\rho>1$ does not hold. Namely $\sum_{k, m=0}^{\infty} \rho^{k-m} \operatorname{cap}^{(\alpha)}\left(E_{k, m}\right)<\infty$. For a compact neighborhood $U$ of $0, \mu$ and $\mu_{k, m}$ denote the associated measures of $R_{C \Omega \cap U}^{(\alpha)} 1$ and of $R_{E_{k, m} \cap U}^{(\alpha)} 1$. respectively. Then by Lemma 3.1, (1), we have

$$
\left.W^{(\alpha)} \mu\right|_{E_{k, m} \cap U} \leqq W^{(\alpha)} \mu_{k, m} \quad \text { on } R^{n+1},
$$

so that

$$
\begin{aligned}
R_{C \Omega \cap U}^{(\alpha)} 1(0) & =W^{(\alpha)} \mu(0) \leqq \sum_{k, m=0}^{\infty} W^{(\alpha)} \mu_{k, m}(0) \\
& =\sum_{k, m=0}^{\infty} \int W^{(\alpha)} \varepsilon_{Y}(0) d \mu_{k, m}(Y) \\
& \leqq C_{0} \rho \sum_{k, m=0}^{\infty} \rho^{k-m} \operatorname{cap}^{(\alpha)}\left(E_{k, m} \cap U\right),
\end{aligned}
$$

where $C_{0}$ is the constant in Remark 4.2. Hence $R_{C \Omega \cap U}^{(\alpha)} 1(0)<1$ for a sufficiently small $U$. This and Proposition 2.12 imply that 0 is not regular.

Assume that 0 is not regular. Denote by $Z$ and $Z^{+}$the totality of integers and that of non-negative integers, respectively. For any $k \in Z^{+}$ and $m \in Z^{+}$with $m \geqq 1$, we put

$$
\begin{aligned}
\Gamma_{k, m}= & \left\{(x,-t) \in R^{n+1} ; 0<t \leqq \rho^{-2 \alpha k / n}, \frac{1}{2} \rho^{m /(n+2 \alpha)-(k+1) / n}\right. \\
& \left.<|x|<\rho^{(m+1) /(n+2 \alpha)-k / n}+\frac{1}{2} \rho^{m /(n+2 \alpha)-(k+1) / n}\right\}
\end{aligned}
$$

and 


$$
\Gamma_{k, 0}=\left\{(x,-t) \in R^{n+1} ; \rho^{-2 \alpha(k+1) / n} \leqq t \leqq \rho^{-2 \alpha k / n}\right\} .
$$

Choose a fixed $j_{0} \in Z^{+}$with $j_{0} \geqq 2$ and $j_{0}>\left((n+2 \alpha)^{2} / 2 \alpha\right) \log _{\rho}\left(d_{1} / d_{2}\right)$, where $d_{1}=\rho^{1 /(n+2 \alpha)+1 / n}+\frac{1}{2}$ and $d_{2}=\frac{1}{2} \rho^{-(1 /(n+2 \alpha)+1 / n)}$. Put

$$
\Lambda_{k, m}=\left\{\left(k+j_{0} p, m+j_{0} q\right) \in Z^{+} \times Z^{+} ; p, q \in Z, A_{k+j_{0} p, m+j_{0} q} \cap \Gamma_{k, m} \neq \phi\right\} .
$$

If $\left(k+j_{0} p, j_{0} q\right) \in \Lambda_{k, 0}$, then $p=0$ and $q \geqq 0$. If $m \geqq 1$ and $\left(k+j_{0} p, m+j_{0} q\right)$ $\in \Lambda_{k, m}$, then $p \geqq 0$ and

$$
(n+2 \alpha) \log _{\rho} d_{2}+2 \alpha j_{0} p / n<j_{0}(q-p)<(n+2 \alpha) \log _{\rho} d_{1}+2 \alpha j_{0} p / n,
$$

so that $q-p \geqq 0$ and for any $j \in Z^{+}$, there exists at most one pair $(p, q)$ $\in Z \times Z$ such that $q-p=j$ and $\left(k+j_{0} p, m+j_{0} q\right) \in \Lambda_{k, m}$, because

$$
(n+2 \alpha) \log _{\rho}\left(\frac{d_{1}}{d_{2}}\right)<\frac{2 \alpha j_{0}}{n} .
$$

Put

$$
A_{1}=\max \left(C_{0} \rho^{2}\left(1-\rho^{-2 \alpha / n}\right)^{-n / 2 \alpha} \phi_{\alpha}(0), 2^{n+2 \alpha} C_{0} C\left(\frac{1}{2} \rho^{-1 / n}\right) \rho^{2+2 \alpha / n}\right),
$$

where $C_{0}$ and $C\left(\frac{1}{2} \rho^{-1 / n}\right)$ are the constants in Remark 4.2 and in Lemma 4.1 for $r_{0}=\frac{1}{2} \rho^{-1 / n}$. Then for any $(k, m) \in Z^{+} \times Z^{+}, Y \in A_{k, m}$ and $X \in$ $\left.\bigcup_{k+j_{0} p \geqq 0, m+j_{0} q \geqq 0}^{(p, q) \in \times Z} A_{k+j_{0} p, m+j_{0} q}\right) \cap C \Gamma_{k, m}$, we have

$$
W^{(\alpha)} \varepsilon_{Y}(X) \leqq A_{1} W^{(\alpha)} \varepsilon_{Y}(0) .
$$

In fact, for any $Y=(y,-s) \in A_{k, m}$ and $X=(x,-t) \in A_{k+j_{0} p, m+j_{0} q} \cap$ $C \Gamma_{k, m}, W^{(\alpha)} \varepsilon_{Y}(X)=0$ if $s \leqq t$. Assume $s>t$. If $m=0$, then $t \leqq \rho^{-2 \alpha\left(k+j_{0}\right) / n}$ $<\rho^{-2 \alpha(k+1) / n} \leqq s$, so that $s-t \geqq \rho^{-2 \alpha(k+1) / n}\left(1-\rho^{-2 \alpha / n}\right)$, and hence

$$
\begin{aligned}
W^{(\alpha)} \varepsilon_{Y}(X) & =(s-t)^{-n / 2 \alpha} \phi_{\alpha}\left((s-t)^{-1 / 2 \alpha}|x-y|\right) \\
& \leqq \rho^{k+1}\left(1-\rho^{-2 \alpha / n}\right)^{-n / 2 \alpha} \phi_{\alpha}(0) \leqq A_{1} W^{(\alpha)} \varepsilon_{Y}(0) .
\end{aligned}
$$

If $m \geqq 1$, then $\rho^{m /(n+2 \alpha)-(k+1) / n} \leqq|y| \leqq \rho^{(m+1) /(n+2 \alpha)-k / n}$, and hence $|x-y| \geqq$ $\frac{1}{2} \rho^{m /(n+2 \alpha)-(k+1) / n}$, so that $(s-t)^{-1 / 2 \alpha}|x-y| \geqq \frac{1}{2} \rho^{-1 / n}$. Therefore Lemma 4.1 and Remark 4.2 give

$$
\begin{aligned}
W^{(\alpha)} \varepsilon_{Y}(X) & =(s-t)^{-n / 2 \alpha} \phi_{\alpha}\left((s-t)^{-1 / 2 \alpha}|x-y|\right) \\
& \leqq C\left(\frac{1}{2} \rho^{-1 / n}\right)(s-t)|x-y|^{-n-2 \alpha} \\
& \leqq 2^{n+2 \alpha} C\left(\frac{1}{2} \rho^{-1 / n}\right) \rho^{1+2 \alpha / n} \rho^{k-m} \\
& \leqq A_{1} W^{(\alpha)} \varepsilon_{Y}(0) .
\end{aligned}
$$


Thus our required inequality holds.

By Propositions 2.12 and 2.13, we choose a compact neighborhood $U$ of 0 such that $A_{1} R_{C \Omega \cap U}^{(\alpha)} 1(0)<1 / 2$. For any $r>0, \mu_{r}$ denotes a positive measure on $R^{n+1}$ defined by

$$
\int f d \mu_{r}=\int_{|x| \geqq r} f(x,-2) d x
$$

for every $f \in C_{K}\left(R^{n+1}\right)$. Then for $|x| \geqq r$ and $t>-2$, we have

$$
W^{(\alpha)} \mu_{r}(x, t)=\int_{|y| \geqq r} W^{(\alpha)}(x-y, t+2) d y>1 / 2,
$$

because

$$
\int_{R^{n}} W^{(\alpha)}(x-y, t+2) d y=\int_{R^{n}} d P_{t+2}^{(\alpha)}=1 .
$$

Since $\lim _{r \rightarrow \infty} W^{(\alpha)} \mu_{r}(0)=0$, we can choose $r>0$ such that $A_{1} R_{T_{r}}^{(\alpha)} 1(0)_{1}<\frac{1}{2}$, where $T_{r}=\left\{(x, t) \in R^{n+1} ;|x| \geqq r, t \geqq-1\right\}$. Hence

$$
1-\mathrm{A}_{1} R_{C \Omega \cap\left(U \cup T_{r}\right)}^{(\alpha)} 1(0) \geqq 1-A_{1}\left(R_{C \Omega \cap U}^{(\alpha)} 1(0)+R_{T_{r}}^{(\alpha)} 1(0)\right)>0
$$

(see Remark 3.4). We take a fixed $(i, j) \in Z^{+} \times Z^{+}$with $i, j<j_{0}$. For $(p, q) \in Z^{+} \times Z^{+}$, we set $k_{p}=j_{0} p+i$ and $m_{q}=j_{0} q+j$. Put

$$
F=\bigcup_{p, q=0}^{\infty}\left(E_{k_{p}, m_{q}} \cap\left(U \cup T_{r}\right)\right) \text { and } F_{p, q}=E_{k_{p}, m_{q}} \cap\left(U \cup T_{r}\right) .
$$

Then for any $(p, q),\left(p^{\prime}, q^{\prime}\right) \in Z^{+} \times Z^{+}$, we have

$$
F_{p^{\prime}, q^{\prime}}=A_{k_{p}+\left(p^{\prime}-p\right) j_{0}, m_{q}+\left(q^{\prime}-q\right) j_{0}} \cap C \Omega \cap\left(U \cup T_{r}\right) \text {. }
$$

For $(p, q) \in Z^{+} \times Z^{+}$, we write

$$
I_{p, q}=\left\{\left(p^{\prime}, q^{\prime}\right) \in Z^{+} \times Z^{+} ;\left(k_{p}, m_{q}\right) \notin \Lambda_{k_{p^{\prime}, m_{q^{\prime}}}}\right\}
$$

and

$$
I_{p, q}^{\prime}=\left\{\left(p^{\prime}, q^{\prime}\right) \in Z^{+} \times Z^{+} ;\left(k_{p}, m_{q}\right) \in \Lambda_{k_{p^{\prime}, m_{q^{\prime}}}},\left(p^{\prime}, q^{\prime}\right) \neq(p, q)\right\} .
$$

For $(p, q) \in Z^{+} \times Z^{+}$, we denote by $\mu_{p, q}$ the associated measure of $R_{F_{p, q}}^{(\alpha)} 1$ and by $\mu$ the associated measure of $R_{F}^{(\alpha)} 1$. By Proposition 2.5, (1), we have $R_{F}^{(\alpha)} 1=W^{(\alpha)} \mu$. For any $(p, q) \in Z^{+} \times Z^{+},\left(p^{\prime}, q^{\prime}\right) \in I_{p, q}$ and any $Y \in F_{p^{\prime}, q^{\prime}}$, we have

$$
W^{(\alpha)} \varepsilon_{Y}(X) \leqq A_{1} W^{(\alpha)} \varepsilon_{Y}(0) \quad \text { on } F_{p, q}
$$


and hence

$$
W^{(\alpha)} \varepsilon_{Y, F_{p, q}^{\prime}}^{\prime} \leqq A_{1} W^{(\alpha)} \varepsilon_{Y}(0) W^{(\alpha)} \mu_{p, q} \quad \text { on } R^{n+1} .
$$

This and Proposition 2.9 give

$$
W^{(\alpha)}\left(\left.\mu\right|_{F_{p^{\prime}, q^{\prime}}}\right)_{F_{p, q}}^{\prime} \leqq A_{1} W^{(\alpha)}\left(\left.\mu\right|_{F_{p^{\prime}, q^{\prime}}}\right)(0) W^{(\alpha)} \mu_{p, q} \quad \text { on } R^{n+1}
$$

Hence, by Proposition 2.9 and Lemma 2.14, we obtain that for any $(p, q)$ $\in Z^{+} \times Z^{+}$

$$
\begin{aligned}
W^{(\alpha)} \mu_{p, q}= & \left.W^{(\alpha)} \mu\right|_{F_{p, q}}+W^{(\alpha)}\left(\mu-\left.\mu\right|_{F_{p, q}}\right)_{F_{p, q}}^{\prime} \\
= & \left.W^{(\alpha)} \mu\right|_{F_{p, q}}+\sum_{\left(p^{\prime}, q^{\prime}\right) \in I_{p, q}} W^{(\alpha)}\left(\left.\mu\right|_{\left.F_{p^{\prime}, q^{\prime}}\right)_{F_{p, q}}^{\prime}}\right. \\
& +\sum_{\left(p^{\prime}, q^{\prime}\right) \in I_{p, q}^{\prime},} W^{(\alpha)}\left(\left.\mu\right|_{F_{p^{\prime}}, q^{\prime}}\right)_{F_{p, q}}^{\prime} \\
\leqq & \left.W^{(\alpha)} \mu\right|_{F_{p, q}}+A_{1} \sum_{\left(p^{\prime}, q^{\prime}\right) \in I_{p, q}} W^{(\alpha)} \mu_{F_{p^{\prime}}, q^{\prime}}(0) W^{(\alpha)} \mu_{p, q} \\
& +\sum_{\left(p^{\prime}, q^{\prime}\right) \in I_{p, q}^{\prime}} W^{(\alpha)}\left(\left.\mu\right|_{F_{p^{\prime}, q^{\prime}}}\right)_{F_{p, q}}^{\prime} \\
\leqq & \left.W^{(\alpha)} \mu\right|_{F_{p, q}}+A_{1} W^{(\alpha)} \mu(0) W^{(\alpha)} \mu_{p, q} \\
& +\sum_{\left(p^{\prime}, q^{\prime}\right) \in I_{p, q}^{\prime}} W^{(\alpha)}\left(\left.\mu\right|_{\left.F_{p^{\prime}, q^{\prime}}\right)_{F_{p, q}}^{\prime}} \text { on } R^{n+1} .\right.
\end{aligned}
$$

Putting $A_{2}=1-A_{1} R_{C \Omega \cap\left(U \cup T_{r}\right)}^{(\alpha)} 1(0)>0$, we have

$$
A_{2} W^{(\alpha)} \mu_{p, q}(0) \leqq\left. W^{(\alpha)} \mu\right|_{F p, q}(0)+C_{0} \sum_{\left(p^{\prime}, q^{\prime}\right) \in I_{p, q}^{\prime}} \int \rho^{k_{p}-m_{q}+1} d\left(\left.\mu\right|_{F_{p^{\prime}}, q^{\prime}}\right)
$$

(see Remark 4.2), because $\operatorname{supp}\left[\left(\left.\mu\right|_{\left.F_{p^{\prime}, q^{\prime}}\right)_{F_{p, q}}^{\prime}}\right] \subset F_{p, q}\right.$ and $\int d\left(\left.\mu\right|_{\left.F_{p^{\prime}, q^{\prime}}\right)_{F_{p, q}}^{\prime} \leqq} \leqq\right.$ $\int_{1} d\left(\left.\mu\right|_{F_{p^{\prime}, q^{\prime}}}\right)$ (see Proposition 2.5, (3) and Corollary 2.8). Therefore

$$
\begin{aligned}
& A_{2} \sum_{p, q=0}^{\infty} W^{(\alpha)} \mu_{p, q}(0) \\
& \leqq W^{(\alpha)} \mu(0)+C_{0} \sum_{p, q=0}^{\infty} \sum_{\left(p^{\prime}, q^{\prime}\right) \in I^{\prime}, q} \int \rho^{k_{p}-m_{q^{+1}}} d\left(\left.\mu\right|_{F_{p^{\prime}, q^{\prime}}}\right) \\
& =W^{(\alpha)} \mu(0)+C_{0} \rho \sum_{p^{\prime}, q^{\prime}=0}^{\infty} \sum_{\substack{\left(k, p, m_{q}\right) \in A \\
(p, q) \neq\left(p^{\prime}, q^{\prime}\right)}}^{\infty}\left(\rho^{k_{p}-m_{q}}\right) \int d\left(\left.\mu\right|_{F_{p^{\prime}}, q^{\prime}}\right) \\
& \leqq W^{(\alpha)} \mu(0)+C_{0} \rho \sum_{p^{\prime}, q^{\prime}=0}^{\infty} \sum_{t=1}^{\infty}\left(\rho^{k_{p^{\prime}}-m_{\mathbf{q}^{\prime}}-j_{0} t}\right) \int d\left(\left.\mu\right|_{F^{\prime} \boldsymbol{p}^{\prime}, q^{\prime}}\right) \\
& \leqq W^{(\alpha)} \mu(0)+\left(C_{0} \rho\right)^{2}\left(\rho^{j 0}-1\right)^{-1} \sum_{p^{\prime}, q^{\prime}=0}^{\infty} W^{(\alpha)}\left(\left.\mu\right|_{F_{p^{\prime}, q^{\prime}}}\right)(0) \\
& =A_{3} W^{(\alpha)} \mu(0) \text {, }
\end{aligned}
$$

where $A_{3}=1+\left(C_{0} \rho\right)^{2}\left(\rho^{j_{0}}-1\right)^{-1}$. Thus Lemma 3.1, (2) and Remark 4.2 give 


$$
\sum_{p, q=0}^{\infty} \rho^{j_{0}(p-q)+i-j} \operatorname{cap}^{(\alpha)}\left(E_{j_{0} p+i, j_{0} q+j} \cap\left(U \cup T_{r}\right)\right) \leqq C_{0} \rho A_{2}^{-1} A_{3} W^{(\alpha)} \mu(0)<\infty .
$$

Since $i, j$ are arbitrary, we have

$$
\sum_{k, m=0}^{\infty} \rho^{k-m} \operatorname{cap}^{(\alpha)}\left(E_{k, m} \cap\left(U \cup T_{r}\right)\right)<\infty .
$$

On the other hand, $\left\{(k, m) \in Z^{+} \times Z^{+} ; E_{k, m} \cap C\left(U \cup T_{r}\right) \neq \phi\right\}$ being finite, we obtain that

$$
\sum_{k, m=0}^{\infty} \rho^{k-m} \operatorname{cap}^{(\alpha)}\left(E_{k, m} \cap C\left(U \cup T_{r}\right)\right)<\infty .
$$

Thus the strong subadditivity of $\operatorname{cap}^{(\alpha)}(K)$ shows that $w_{\rho, p}<\infty$. This completes the proof of our main theorem.

Corollary 4.3. Let $\Omega$ be an open set in $R^{n+1}, \rho>1$ and $X_{0}$ a boundary point of $\Omega$. Then $X_{0}$ is regular if and only if the following condition (w') holds:

$$
\sum_{k=0}^{\infty} \rho^{k} \operatorname{cap}^{(\alpha)}\left(A_{k}\left(X_{0}\right) \cap C \Omega\right)=\infty,
$$

where $A_{k}\left(X_{0}\right)=\left\{X \in R^{n+1} ; \rho^{k} \leqq W^{(\alpha)}\left(X_{0}-X\right) \leqq \rho^{k+1}\right\}$.

Proof. By the subadditivity of $\operatorname{cap}^{(\alpha)}(K)$, we have $w_{\rho, \rho}=\infty$ provided that $\left(\mathrm{w}^{\prime}\right)$ holds, and hence by our theorem, the "if" part holds. The "only if" part is proved in the same manner as in the proof of the "only if" part of our theorem.

By generalizing a Poincaré type condition for regular points given in [6], we have the following

Corollary 4.4. Let $\Omega$ be an open set in $R^{n+1}$ and $X_{0}=\left(x_{0}, t_{0}\right) a$ boundary point of $\Omega$. If there exists a Borel set $B$ in $R^{n}$ with positive Lebesgue measure whose $\alpha$-tusk $T_{X_{0}}^{(\alpha)}(B)$ at $X_{0}$ is contained in $C \Omega$, then $X_{0}$ is regular for the Dirichlet problem of $L^{(\alpha)}$ on $\Omega$.

The $\alpha$-tusk $T_{X_{0}}^{(\alpha)}(B)$ of $B$ at $X_{0}=\left(x_{0}, t_{0}\right)$ is the set $\left\{\left(x_{0}+p x, t_{0}-p^{2 \alpha}\right)\right.$; $\left.x \in B, 0<p \leqq p_{0}\right\}$ for some $p_{0}>0$.

Proof. We may assume $X_{0}=0$ and $p_{0}=1$. Choose $\rho>1$ and an integer $m_{0} \geqq 1$ such that the Lebesgue measure of $B \cap\left\{x \in R^{n} ;(x,-1) \in\right.$ $\left.A_{0, m_{0}}\right\}$ is positive, where $A_{k, m}$ is the set in $R^{n+1}$ defined in our theorem for $\lambda=\rho$ and $\rho\left(k, m \in Z^{+}\right)$. For the simplicity of the notation, we put $B^{\prime}=$ 
$B \cap\left\{x \in R^{n} ;(x,-1) \in A_{0, m_{0}}\right\}$ and $A_{k, m_{0}}^{\prime}=T_{X_{0}}^{(\alpha)}(B) \cap A_{k, m_{0}}\left(k \in Z^{+}\right)$. By Remark 3.7, we have

$$
\operatorname{cap}^{(\alpha)}\left(A_{0, m_{0}}\right) \geqq \operatorname{cap}^{(\alpha)}\left(\left\{(x,-1) ; x \in B^{\prime}\right\}\right)>0 .
$$

Since $\tau_{\rho-k / n}\left(A_{0, m_{0}}^{\prime}\right)=A_{k, m_{0}}^{\prime}$ for every $k \geqq 1$, Proposition 3.6 gives $\operatorname{cap}^{(\alpha)}\left(A_{k, m_{0}}^{\prime}\right)$ $=\rho^{-k} \operatorname{cap}^{(\alpha)}\left(A_{0, m_{0}}^{\prime}\right)$, which implies that $w_{\rho, \rho}=\infty$. Thus our theorem shows Corollary 4.4 .

In [6], we proved an analogous assertion for a nonempty open set in place of $B$.

Remark 4.5. It is an open question if the Wiener criterion of type (w) for $(\partial / \partial t)-\Delta$ holds.

\section{REFERENCES}

[1] C. Berg and G. Forst, Potential theory on locally compact abelian groups, SpringerVerlag, Berlin Heiderberg New York, 1975.

[2] G. Choquet, Lectures on analysis, vol. I, Benjamin, New York, 1969.

[ 3 ] E. Effros and J. Kazdan, On the Dirichlet problem for the heat equation, Indiana Univ. Math. J., 20 (1971), 683-693.

[4] L. Evans and R. Gariepy, Wiener's criterion for the heat equation, Arch. Rational Mech. Anal., 78 (1982), 293-314.

[ 5 ] L. L. Helms, Introduction to potential theory, Wiley-Interscience, New York, 1969.

[ 6 ] M. Itô and M. Nishio, Poincaré type conditions of the regularity for the parabolic equation of order $\alpha$, Nagoya Math. J., 115 (1989), 1-22.

[ 7 ] N. A. Watson, Thermal capacity, Proc. London Math. Soc., 37 (1978), 342-362.

Department of Mathematics

School of Science

Nagoya University

Chikusa-ku, Nagoya, 464-01

Japan 